\title{
Treatment of Refractory Anti-HMG-CoA Reductase Myopathy: A Role for Rituximab?
}

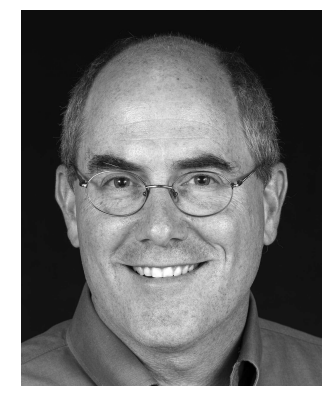

Anti-hydroxymethylglutaryl CoA reductase (HMGCR) myopathy is a subtype of myositis characterized by proximal muscle weakness, elevated serum creatine kinase (CK) levels, and autoantibodies recognizing $\mathrm{HMGCR}^{1}$. While statins are an established risk factor for developing anti-HMGCR myopathy in older patients, some individuals develop this condition without a known statin exposure ${ }^{2,3}$. To date, effective treatment strategies have not been established in clinical trials. Nonetheless, many patients with anti-HMGCR myopathy improve with immunosuppressive therapy, and current expert opinion guidelines recommend initiating treatment with corticosteroids, methotrexate, and/or intravenous immunoglobulin (IVIG) ${ }^{1}$. Unfortunately, a significant number of patients with anti-HMGCR myopathy have persistently active disease despite aggressive treatment with these and other agents. Indeed, a study including 50 patients with anti-HMGCR myopathy treated for 2 years or more found that $30 \%$ continued to have weakness and elevated muscle enzymes ${ }^{4}$. This underscores the importance of finding more effective treatment modalities for these patients.

A number of observations suggest the possibility that autoantibodies may play a pathogenic role in anti-HMGCR myopathy. For example, anti-HMGCR titers have been shown to correlate with disease activity ${ }^{4}$. In addition, muscle biopsies from patients with anti-HMGCR myopathy reveal membrane attack complex deposited on the surface of muscle fibers ${ }^{5}$. Further, anti-HMGCR autoantibodies not only cause atrophy of cultured human myotubes ${ }^{6}$, but they also lead to myofiber necrosis (albeit limited) and muscle weakness when injected into mice ${ }^{7}$. Given the possibility that anti-HMGCR autoantibodies may be pathogenic and given the prominent role played by B cells in autoantibody production, B cell depletion would appear to be an effective treatment strategy for anti-HMGCR myopathy.

In this issue of The Journal, Landon-Cardinal, et al ${ }^{8}$ report on the outcomes of 9 patients with anti-HMGCR myopathy who were treated with rituximab (RTX), a chimeric monoclonal antibody targeting CD20+ B cells. These patients had disease durations ranging from 0.75 to 23 years and refractory disease despite prior treatment with 1 or more agents, often including IVIG. While 6 of these patients had no clear improvement with RTX, 3 had a positive response defined by (1) a reduction of CK levels to less than twice the upper limit of normal and/or resolution of T2/ short-tau inversion recovery hyperintensities on magnetic resonance imaging (MRI), (2) no worsening of proximal muscle strength, and (3) the tapering of corticosteroids to $<15 \mathrm{mg}$ per day and the discontinuation of IVIG. None of the RTX responders had a known statin exposure.

Along with a previously published case series reporting improvement in 2 out of 5 patients with anti-HMGCR myopathy treated with $\mathrm{RTX}^{9}$, the current study provides additional evidence that $\mathrm{B}$ cell depletion may be effective in patients with refractory disease. However, several observations may limit optimism about the efficacy of RTX in the patients described by Landon-Cardinal and colleagues ${ }^{8}$. The first responder was simultaneously treated with prednisone, IVIG, and cyclophosphamide, making it difficult to assess whether RTX was responsible for the improvement. The second and third responders had disease for more than 10 years and were severely weak with normal CK levels prior to RTX therapy. Because high CK levels are usually associated with disease activity in anti-HMGCR myopathy, these 2 patients either had relatively inactive disease or extensive fatty replacement of muscle tissue that prevented an elevated CK level. In the second responder, a change in strength from 2 to 3 out of 5 on the Medical Research Council strength scale was the sole indicator of response. The third responder had no change in strength or CK levels with RTX, and only the resolution of muscle edema on MRI suggested any response to the treatment. While a modest improvement in strength and the resolution of edema on MRI are encouraging, one would hope to see more robust evidence of treatment response before concluding with certainty that RTX is an effective therapy for some patients

See Rituximab for anti-HMGCR myopathy, page 623

Personal non-commercial use only. The Journal of Rheumatology Copyright @ 2019. All rights reserved. 
with anti-HMGCR myopathy. Such evidence will most likely emerge only from a well-designed clinical trial of RTX in this patient population.

The limitations of this retrospective case series notwithstanding, the observations of Landon-Cardinal, et $a l^{8}$ suggest that RTX could be considered as a rescue therapy in patients with refractory anti-HMGCR myopathy. Assuming some patients with anti-HMGCR myopathy do respond to RTX, the study also raises questions. If given earlier, could RTX have prevented the extensive chronic muscle damage presumably experienced by 2 of the responders? How many other agents should be tried and for how long before resorting to RTX? Why do only some patients seem to respond to RTX? What treatments should we offer the majority of patients with refractory anti-HMGCR myopathy who do not respond to RTX, IVIG, and other conventional immunosuppressive medications? Given the deposition of membrane attack complex on the surface of myofibers, could inhibitors of the terminal complement pathway be considered as a possible treatment option? These are pressing questions for the one-third of patients with anti-HMGCR myopathy who currently have a chronic progressive myopathic process despite aggressive immunosuppressive therapy.

ANDREW L. MAMMEN, MD, PhD, Muscle Disease Unit, (US) National Institute of Arthritis and Musculoskeletal and Skin Diseases, National Institutes of Health, Bethesda, Maryland, USA.

Address correspondence to Dr. A.L. Mammen, National Institutes of Health, 50 South Drive, Room 1141, Building 50, MSC 8024, Bethesda, Maryland 20892, USA. E-mail: andrew.mammen@nih.gov. This work was funded by the Intramural Research Programs of the US National Institute of Arthritis and Musculoskeletal and Skin Diseases of the US National Institutes of Health. Dr. Mammen holds a patent on a commercial test for anti-HMGCR autoantibodies, but receives no financial compensation for this.

\section{REFERENCES}

1. Allenbach Y, Mammen AL, Benveniste O, Stenzel W, Immune-Mediated Necrotizing Myopathies Working Group. 224th ENMC international workshop:: Clinico-sero-pathological classification of immune-mediated necrotizing myopathies. Zandvoort, the Netherlands, 14-16 October 2016. Neuromuscul Disord 2018;28:87-99.

2. Mammen AL, Chung T, Christopher-Stine L, Rosen P, Rosen A, Doering KR, et al. Autoantibodies against 3-hydroxy-3-methylglutaryl-coenzyme a reductase in patients with statin-associated autoimmune myopathy. Arthritis and Rheumatism 2011;63:713-21.

3. Watanabe Y, Uruha A, Suzuki S, Nakahara J, Hamanaka K, Takayama K, et al. Clinical features and prognosis in anti-SRP and anti-HMGCR necrotising myopathy. J Neurol Nurosurg Psychiatry 2016;87:1038-44.

4. Tiniakou E, Pinal-Fernandez I, Lloyd TE, Albayda J, Paik J, Werner JL, et al. More severe disease and slower recovery in younger patients with anti-3-hydroxy-3-methylglutaryl-coenzyme a reductase-associated autoimmune myopathy. Rheumatology 2017;56:787-94.

5. Christopher-Stine L, Casciola-Rosen LA, Hong G, Chung T, Corse AM, Mammen AL. A novel autoantibody recognizing 200-kd and $100-\mathrm{kd}$ proteins is associated with an immune-mediated necrotizing myopathy. Arthritis Rheum 2010;62:2757-66.

6. Arouche-Delaperche L, Allenbach Y, Amelin D, Preusse C, Mouly $\mathrm{V}$, Mauhin W, et al. Pathogenic role of anti-signal recognition protein and anti-3-Hydroxy-3-methylglutaryl-CoA reductase antibodies in necrotizing myopathies: Myofiber atrophy and impairment of muscle regeneration in necrotizing autoimmune myopathies. Ann Neurol 2017;81:538-48.

7. Bergua C, Chiavelli H, Allenbach Y, Arouche-Delaperche L, Arnoult $\mathrm{C}$, Bourdenet $\mathrm{G}$, et al. In vivo pathogenicity of $\mathrm{IgG}$ from patients with anti-SRP or anti-HMGCR autoantibodies in immune-mediated necrotising myopathy. Ann Rheum Dis 2018 Oct 11 (E-pub ahead of print).

8. Landon-Cardinal O, Allenbach Y, Soulages A, Rigolet A, Hervier B, Champtiaux $\mathrm{N}$, et al. Rituximab in the treatment of refractory anti-HMGCR immune-mediated necrotizing myopathy. J Rheumatol 2019;46:623-7.

9. Ashton C, Junckerstorff R, Bundell C, Hollingsworth P, Needham $\mathrm{M}$. Treatment and outcomes in necrotising autoimmune myopathy: An Australian perspective. Neuromuscul Disord 2016;26:734-40.

J Rheumatol 2019;46:550-1; doi:10.3899/jrheum.181392

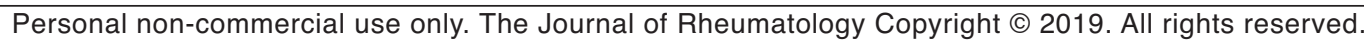

